\title{
A GEOMETRIC DESCRIPTION OF THE SETS OF PALINDROMIC AND ALTERNATING MATRIX PENCILS WITH BOUNDED RANK*
}

\author{
FERNANDO DE TERÁN ${ }^{\dagger}$
}

\begin{abstract}
The sets of $n \times n$ T-palindromic, T-antipalindromic, T-even, and T-odd matrix pencils with rank at most $r<n$ are algebraic subsets of the set of $n \times n$ matrix pencils. In this paper, we determine their dimension and we prove that they are all irreducible. This is in contrast with the nonstructured case, since it is known that the set of $n \times n$ matrix pencils with rank at most $r<n$ is an algebraic set with $r+1$ irreducible components. We also show that these sets of structured pencils with bounded rank are the closure of the congruence orbit of a certain structured pencil given in canonical form. This allows us to determine the generic canonical form of a structured $n \times n$ matrix pencil with rank at most $r$, for any of the previous structures.
\end{abstract}

Key words. matrix pencil, T-palindromic, T-alternating, strict equivalence, congruence, orbit, spectral information, algebraic set

AMS subject classifications. 15A22, 15A18, 15A21, 65F 15

DOI. $10.1137 / 17 \mathrm{M} 1124735$

1. Introduction. Structured matrix pencils arise in several problems of applied mathematics, either as modeling physical systems by themselves or as a tool to compute the relevant information in the analysis of other higher-order systems by linearization (see, for instance, [22] and the references therein). Some of the structures appearing most frequently in applications are the skew-symmetric, the T-palindromic (or T-antipalindromic), and the T-alternating structures (see section 2 for the definition of these structures). Among the sets of $n \times n$ structured matrix pencils, an important class from the point of view of applications is the class of low-rank pencils. In this paper, low-rank means essentially rank-deficient, that is, we are interested in $n \times n$ pencils with rank $r$ and $r<n$. Low-rank pencils arise when modeling systems that depend on many parameters, but only a few of them are modified (or perturbed), regardless of the size (in norm) of the modification. Some particular settings where low-rank pencils arise include dissipative dynamical systems [2, sect. 1.2], network analysis in electrical engineering [27], or multibody system simulation [18].

Therefore, low-rank matrix pencils naturally arise associated with low-rank perturbations, a subject which has attracted the attention of researchers in the recent years $[1,2,4,7,9,24,25]$. In a similar way as the understanding of the underlying geometry of the set of matrix pencils is helpful to analyze the change of the scalar spectral information under small perturbations $[16,17]$, the analysis of the geometry of low-rank matrix pencils may be helpful in the explanation of the change of the spectral information under low-rank perturbations. To be more precise, the scalar spectral information (partial multiplicities and minimal indices) comprises the invariants of matrix pencils under strict equivalence transformations. Two matrix pencils

* Received by the editors April 7, 2017; accepted for publication (in revised form) by F. Tisseur April 19, 2018; published electronically July 3, 2018.

http://www.siam.org/journals/simax/39-3/M112473.html

Funding: This work was supported by the Ministerio de Economía y Competitividad of Spain through grants MTM2015-68805-REDT and MTM2015-65798-P, and by the Ministerio de Educación, Cultura y Deportes of Spain through grant PRX16/00128 "Programa de estancias de movilidad de profesores e investigadores en centros de enseñanza superior e investigación 'Salvador de Madariaga.' "

†Departamento de Matemáticas, Universidad Carlos III de Madrid, 28911 Leganés, Spain (fteran@ math.uc3m.es). 
$A_{0}+\lambda A_{1}$ and $B_{0}+\lambda B_{1}$ are said to be strictly equivalent if there exist two nonsingular matrices $V, W$ such that $V\left(A_{0}+\lambda A_{1}\right) W=B_{0}+\lambda B_{1}$. Then, all matrix pencils having the same scalar spectral information lie in the strict equivalence orbit of a given pencil $A_{0}+\lambda A_{1}$. As a consequence, the knowledge of the geometry of matrix pencils, in terms of the inclusion relationships between the closures of strict equivalence orbits, may allow to explain the changes in the spectral information due to small perturbations. This is translated to the set of low-rank matrix pencils and low-rank perturbations.

A description of the set of low-rank pencils (nonstructured) was provided in [4], where it was proved that the set of $m \times n$ matrix pencils with rank at most $r<$ $\min \{m, n\}$ is an algebraic set with $r+1$ irreducible components, which are the closures of the equivalence orbits of some specific pencils given in Kronecker canonical form (a similar description has been recently obtained in [11, Thm. 3.2] for the set of $m \times n$ matrix polynomials with grade $d$ and rank at most $r$ ). Another description of the set of $m \times n$ matrix pencils with rank at most $r<\min \{m, n\}$ was presented in the recent papers $[7,8]$. In [7, Lemma 3.1], the authors provide a decomposition of the set of $n \times n$ matrix pencils with rank at most $r$ as the union of $r+1$ sets consisting of sums of $r$ rank-1 pencils in such a way that some of the column vectors of this sum are constant vectors, and the remaining ones are allowed to have degree 1 (though in [7] it is only stated for square pencils, such a decomposition is valid also for rectangular $m \times n$ pencils with rank $r<\min \{m, n\})$. Then, it was proved in [8] that each of these sets corresponds to each of the irreducible components of the algebraic set of matrix pencils with rank at most $r$, which results in a more constructible description of these irreducible components.

If we restrict ourselves to structured pencils and, accordingly, to structured perturbations, some remarkable differences arise. The restrictions imposed by the structure may lead to a different generic behavior under low-rank perturbations [10]. This is just an indication that the analysis of the set of structured low-rank pencils deserves some special attention. In the recent years, some effort has been devoted to analyze and describe the geometry of structured matrix pencils. In particular, the set of skewsymmetric pencils was studied in detail in [13, 14], and the set of symmetric pencils was analyzed in [15]. However, no special attention has been paid so far to the set of structured matrix pencils with bounded rank. To our knowledge, the only reference on this is the recent work [12]. In that paper, the authors describe the generic scalar spectral information of skew-symmetric matrix polynomials with bounded rank using orbit closures. This includes the case of skew-symmetric matrix pencils. The main results in the present work are the counterpart of those in [12], but for $\top$-palindromic, $T$-antipalindromic, and $T$-alternating matrix pencils, instead of skew-symmetric ones.

The set of $n \times n$ structured matrix pencils with rank at most $r<n$, denoted by $\mathbb{S}_{r}$, for any of the structures in Table 2.1, is an algebraic subset of the set of pencils $A_{0}+\lambda A_{1}$, which can be identified with $\mathbb{C}^{2 n^{2}}$ (by considering a pencil $A_{0}+\lambda A_{1}$ as a pair $\left.\left(A_{0}, A_{1}\right)\right)$. To see this, notice that $\mathbb{S}_{r}$ is defined as the intersection of two algebraic sets. The first one is the set defined by the specific structure, and the second one is the set defined by the low-rank condition. Both them are algebraic sets because they can be defined in terms of multivariable polynomials in the entries of the pencil; in other words, in terms of multivariable polynomials with $2 n^{2}$ variables (the $n^{2}$ coordinates of $A_{0}$ and the $n^{2}$ coordinates of $A_{1}$ ). For the second set, these polynomials are all the $(r+1) \times(r+1)$ minors of general $n \times n$ pencils. For the first set, the polynomials depend on the structure. For instance, for the T-palindromic structure, they are $\left[A_{0}\right]_{i j}-\left[A_{1}\right]_{j i}$, for $1 \leq i, j \leq n$. The goal of this paper is to analyze the geometry of $\mathbb{S}_{r}$ and, in particular, to answer the following questions: 
Q1: Which are the irreducible components of $\mathbb{S}_{r}$ ?

Q2: Which is the dimension of $\mathbb{S}_{r}$ ?

One of the motivations to address questions $\mathbf{Q 1}$ and $\mathbf{Q 2}$ is to answer the following question: Given $r<n$, which is the most likely Kronecker canonical form for structured $n \times n$ pencils with rank at most $r$ ? This question is interesting in the context of low-rank perturbations, when one is interested in describing the most likely (generic) change of the Kronecker canonical form due to low-rank perturbations. In particular, for the unstructured case, as mentioned above, the set of $n \times n$ pencils with rank at most $r<n$ is decomposed into $r+1$ sets in [7] (which coincide with the irreducible components of the algebraic set of $n \times n$ pencils with rank at most $r<n$ [8]). This allowed one to analyze the generic change of the Kronecker canonical form of matrix pencils after low-rank perturbations by looking at the behavior when perturbing with pencils in each of these $r+1$ sets.

We provide answers to questions $\mathbf{Q 1}$ and $\mathbf{Q 2}$ and, as a consequence, we determine the most likely (generic) canonical form under congruence of structured matrix pencils with bounded rank, for any of the structures in Table 2.1. Two matrix pencils $A_{0}+\lambda A_{1}$ and $B_{0}+\lambda B_{1}$ are said to be congruent if there is some invertible matrix $P$ such that $B_{0}+\lambda B_{1}=P\left(A_{0}+\lambda A_{1}\right) P^{\top}$. This relation preserves any of the previous structures; that is, if one of $A_{0}+\lambda A_{1}$ or $B_{0}+\lambda B_{1}$ satisfies any of these structures, then the other one does as well. This is no longer true if we replace congruence by strict equivalence, which is the natural relation for unstructured matrix pencils. This is the reason for considering the more restrictive relation of congruence, instead of strict equivalence, in this work. The most remarkable difference with the unstructured case regarding Q1 is that, while the set of $n \times n$ pencils (nonstructured) with rank at most $r<n$ has, as mentioned, $r+1$ different irreducible components, the set $\mathbb{S}_{r}$ is irreducible, so it has only one irreducible component, for any of the structures $\mathbb{S}$ in Table 2.1. And regarding the generic canonical form, while in the unstructured case there is no a generic canonical form for $n \times n$ pencils with rank at most $r$, in the structured case there is such a generic canonical form.

The paper is organized as follows. In section 2 we introduce the basic notation and definitions used in the paper. In section 3 we present a couple of results which are key in the proof of the main results of the paper. These results are provided in section 4. In particular, Theorem 4.1 shows that the set of $n \times n$ T-palindromic pencils with rank at most $r<n$ is an irreducible algebraic set, and provides a description of this set as the closure of the congruence orbit of certain pencil given in canonical form. This is, precisely, the generic canonical form for $T$-palindromic pencils with rank at most $r$, as stated in Corollary 4.3. Similar results are presented in Theorem 4.2 for the T-antipalindromic structure, and in Theorems 4.5 and 4.6 for the T-even and T-odd structures, respectively. In section 4.3 we compare our results on the dimension of the sets of structured pencils with bounded rank with the dimension of the set of arbitrary $n \times n$ structured pencils (that is, when $r=n$, which allows for full-rank pencils), and also with the set of unstructured pencils. We conclude in section 5 with a summary of the main contributions of the paper and some indications on further research on the topic.

2. Basic definitions and notation. Throughout the paper we use the following notation. The symbol $I_{k}$ (or just $I$, when the size is clear by the context) denotes the $k \times k$ identity matrix. By $e_{j}$ we denote the $j$ th canonical vector, that is the $j$ th column of the identity matrix, where the size of this matrix depends on the context. The notation $A^{\top}$ stands for the transpose of the matrix $A$. The set of vector 
TABLE 2.1

Structured pencils and notation for the sets of structured pencils with rank $\leq r$.

\begin{tabular}{|c|c|c|}
\hline Structure set $\mathbb{S}$ & Definition & Notation \\
& $A_{0}+\lambda A_{1} \in \mathbb{S}$ & $\left\{A_{0}+\lambda A_{1} \in \mathbb{S}: \operatorname{rank}\left(A_{0}+\lambda A_{1}\right) \leq r\right\}$ \\
\hline \hline T-palindromic & $A_{1}^{\top}=A_{0}$ & $\operatorname{Pal}_{r}$ \\
T-antipalindromic & $A_{1}^{\top}=-A_{0}$ & $\operatorname{Apal}_{r}$ \\
T-even & $A_{0}^{\top}=A_{0}, A_{1}^{\top}=-A_{1}$ & $\operatorname{Even}_{r}$ \\
T-odd & $A_{0}^{\top}=-A_{0}, A_{1}^{\top}=A_{1}$ & $\operatorname{Odd}_{r}$ \\
\hline
\end{tabular}

polynomials with $n$ coordinates (that is, vectors whose $n$ coordinates are polynomials in the variable $\lambda$ ) is denoted by $\mathbb{C}[\lambda]^{n}$. Given a vector $v(\lambda) \in \mathbb{C}[\lambda]^{n}$, we denote by $\operatorname{deg} v$ the maximum degree of its coordinates. A matrix pencil is a matrix polynomial with degree 1. In other words, a matrix pencil is of the form $A_{0}+\lambda A_{1}$, with $A_{0}, A_{1} \in \mathbb{C}^{m \times n}$. Since we deal only with structured pencils, which must be square, we only consider the case $m=n$. The reversal of $A_{0}+\lambda A_{1}$ is the pencil $\operatorname{rev}\left(A_{0}+\lambda A_{1}\right):=A_{1}+\lambda A_{0}$. The rank of a matrix pencil $A_{0}+\lambda A_{1}$ is the order of the largest nonidentically zero minor (considered as a scalar polynomial in $\lambda$ ). In other words, it is the rank of $A_{0}+\lambda A_{1}$ viewed as a matrix in the field of rational functions in the variable $\lambda$. This is sometimes referred to in the literature as the normal rank (see, for instance, [17]).

We deal with the set of $n \times n$ structured matrix pencils $A_{0}+\lambda A_{1}$ having any of the structures indicated in Table 2.1, and with rank at most $r<n$. This table includes also the abbreviations used in the paper for each of the sets of low-rank structured matrix pencils. The T-even and T-odd structures are both gathered under the common denomination $T$-alternating.

The canonical form under strict equivalence of matrix pencils is the Kronecker canonical form (KCF), which consists of a direct sum of certain (canonical) blocks [19, Ch. XII, sect. 4]. When the pencil enjoys some particular structure, like the ones in Table 2.1, this structure is translated into the KCF in terms of some restrictions in the number and sizes of certain types of blocks. However, the strict equivalence transformations that lead to the KCF do not necessarily preserve the structure. Moreover, the KCF does not fulfill any of the structures in Table 2.1 for general structured pencils. Nonetheless, an equivalent canonical form to the KCF can be achieved using structure-preserving transformations. In particular, for the structures in Table 2.1, these are congruence transformations. In other words, given any pencil $S(\lambda)$ fulfilling any of the structures in Table 2.1, there is a nonsingular matrix $V$ such that $V S(\lambda) V^{\top}$ is in some appropriate canonical form, which depends on the particular structure, and that displays the information contained in the KCF. We will recall later in this section the canonical form for the T-palindromic structure, which is the only one we need in this paper. For the remaining structures, we refer the reader to [2] and the references therein (see also [23, Cor. 4.3] for the $T$-alternating structure). All these canonical forms are, like the KCF, a direct sum of canonical blocks, including some of the following ones.

A right singular block of order $\alpha$ is the $\alpha \times(\alpha+1)$ matrix pencil:

$$
L_{\alpha}(\lambda):=\left[\begin{array}{cccc}
\lambda & 1 & & \\
& \ddots & \ddots & \\
& & \lambda & 1
\end{array}\right]_{\alpha \times(\alpha+1)}
$$


From this block we construct the following four kinds of blocks of size $(2 \alpha+1) \times(2 \alpha+1)$, which appear in the canonical form for structured matrix pencils:

$$
\begin{aligned}
M_{\alpha}^{\sharp}(\lambda) & :=\left[\begin{array}{cc}
0 & L_{\alpha}(\lambda) \\
\operatorname{rev} L_{\alpha}(\lambda)^{\top} & 0
\end{array}\right]_{(2 \alpha+1) \times(2 \alpha+1)}, \\
M_{\alpha}^{-\sharp}(\lambda) & :=\left[\begin{array}{cc}
0 & L_{\alpha}(\lambda) \\
-\operatorname{rev} L_{\alpha}(\lambda)^{\top} & 0
\end{array}\right]_{(2 \alpha+1) \times(2 \alpha+1)}, \\
M_{\alpha}^{b}(\lambda) & :=\left[\begin{array}{cc}
0 & L_{\alpha}(\lambda) \\
L_{\alpha}(-\lambda)^{\top} & 0
\end{array}\right]_{(2 \alpha+1) \times(2 \alpha+1)}, \\
M_{\alpha}^{-b}(\lambda) & :=\left[\begin{array}{cc}
0 & L_{\alpha}(\lambda) \\
-L_{\alpha}(-\lambda)^{\top} & 0
\end{array}\right]_{(2 \alpha+1) \times(2 \alpha+1)} .
\end{aligned}
$$

More precisely, the $T$-palindromic canonical form contains blocks of type $M_{\alpha}^{\sharp}(\lambda)$, the T-antipalindromic canonical form contains blocks of type $M_{\alpha}^{-\sharp}(\lambda)$, the T-even canonical form contains blocks of type $M_{\alpha}^{b}(\lambda)$, and the $T$-odd canonical form contains blocks of type $M_{\alpha}^{-b}(\lambda)$. These are the blocks associated to the singular spectral structure. The canonical forms contain also a direct sum of blocks that comprise the regular spectral structure, and which are built up from Jordan blocks associated with finite and infinite eigenvalues. A Jordan block of order $k$ associated with the eigenvalue $\lambda_{0}$ is the following block with size $k \times k$ :

$$
J_{k}\left(\lambda_{0}\right)=\left[\begin{array}{cccc}
\lambda_{0} & 1 & & \\
& \ddots & \ddots & \\
& & \lambda_{0} & 1 \\
& & & \lambda_{0}
\end{array}\right]_{k \times k}
$$

Now we are in the position to state the canonical form for congruence of $T$-palindromic matrix pencils. Such a canonical form can be found in [29], but we are using here a different notation. For more details on this canonical form we refer the reader to [3].

Theorem 2.1. (Canonical form of $\top$-palindromic pencils). Any $\top$-palindromic matrix pencil $L(\lambda)$ is congruent to a direct sum of blocks of the following types:

(i) Blocks of type $M_{\alpha}^{\sharp}(\lambda)$.

(ii) Palindromic pairs of Jordan-like blocks with even size associated with $\lambda_{0}=-1$ :

$$
\left[\begin{array}{cc}
0 & \lambda I_{\beta}+J_{\beta}(1) \\
I_{\beta}+\lambda J_{\beta}(1)^{\top} & 0
\end{array}\right],
$$

with $\beta$ an even number.

(iii) Palindromic pairs of Jordan-like blocks with odd size associated with $\lambda_{0}=1$ :

$$
\left[\begin{array}{cc}
0 & \lambda I_{\gamma}+J_{\gamma}(-1) \\
I_{\gamma}+\lambda J_{\gamma}(-1)^{\top} & 0
\end{array}\right],
$$

with $\gamma$ an odd number.

(iv) Palindromic pairs of Jordan-like blocks associated with $-\lambda_{0}$ and $-1 / \lambda_{0}$ $\left(\lambda_{0} \neq \pm 1\right)$ :

$$
\left[\begin{array}{cc}
0 & \lambda I_{\delta}+J_{\delta}\left(\lambda_{0}\right) \\
I_{\delta}+\lambda J_{\delta}\left(\lambda_{0}\right)^{\top} & 0
\end{array}\right]
$$


(v) Palindromic Jordan-like blocks with even size associated with $\lambda_{0}=1$ :

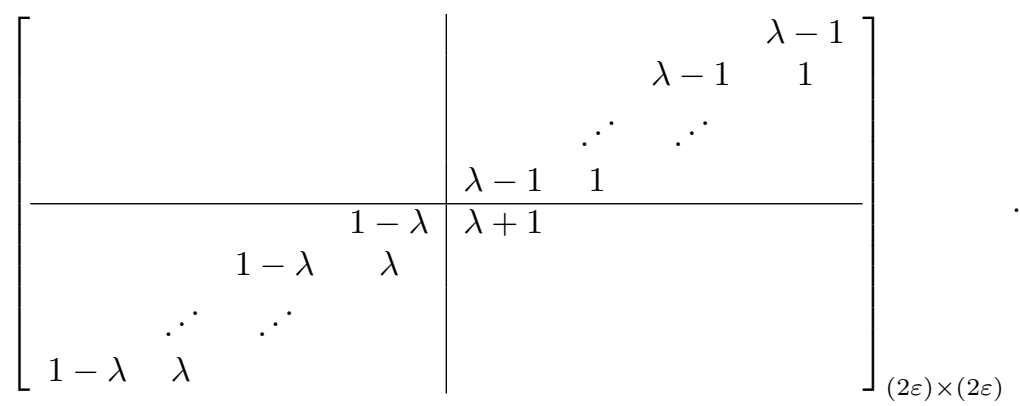

(vi) Palindromic Jordan-like blocks with odd size associated with $\lambda_{0}=-1$ :

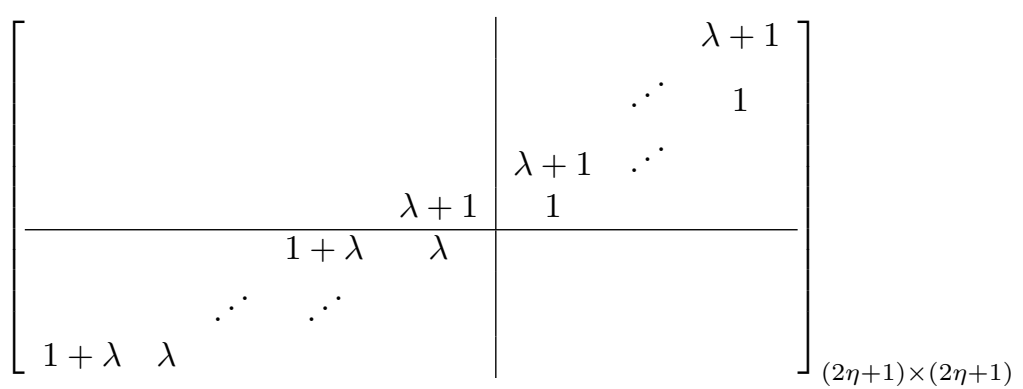

The number of blocks of each type and their particular sizes uniquely depend on $L(\lambda)$ and determine its $\top$-palindromic canonical form.

The $T$-palindromic canonical form in Theorem 2.1 is closely related to the canonical form for congruence (CFC) of matrices, so that there is a one-to-one correspondence between blocks in these canonical forms [3, Thm. 4]. The only relevant correspondence in our developments is the one between so-called type 0 blocks, which are Jordan blocks associated with $\lambda_{0}=0$ (see [3, Thm. 3]), and blocks of type (i) in Theorem 2.1. More precisely, for each block $J_{2 \alpha+1}(0)$ in the CFC of a matrix $A$ there is a block $M_{\alpha}^{\sharp}(\lambda)$ in the $\top$-palindromic canonical form of $A+\lambda A^{\top}$, and vice versa [3, Thm. 4-(ii)]. This correspondence will be used in the proof of Theorem 4.1.

Another relevant notion in this paper is the orbit under congruence of a matrix pencil $L(\lambda)$, which is defined as

$$
\mathcal{O}_{c}(L)=\left\{V^{\top} L(\lambda) V: \quad V \text { nonsingular }\right\}
$$

The closure of this orbit, denoted by $\overline{\mathcal{O}}_{c}(L)$, is the closure in the standard topology, which is the same as the closure in the Zariski topology [26, Thm. 2.33].

Definition 2.2. Let $\mathbb{S}_{r}$ be the set of $n \times n$ structured matrix pencils with rank at most $r$, with $\mathbb{S}$ being any of the structures in Table 2.1. We say that a particular matrix $K_{\mathbb{S}}(\lambda)$ given in structured canonical form is the generic canonical form in $\mathbb{S}_{r}$ if there is a dense open set of $n \times n$ matrix pencils in $\mathbb{S}_{r}$ which are congruent to $K_{\mathbb{S}}(\lambda)$.

In other words, $K_{\mathbb{S}}(\lambda)$ is the generic canonical form in $\mathbb{S}_{r}$ if $\overline{\mathcal{O}}_{c}\left(K_{\mathbb{S}}\right)=\mathbb{S}_{r}$, since $\mathcal{O}_{c}\left(K_{\mathbb{S}}\right)$ is an open set in its closure [21, p. 60].

3. Preliminary results. We first present a decomposition of a given $n \times n \top$ palindromic matrix pencil with rank at most $r$ as the sum of $r$ rank-1 pencils. This decomposition provides us a constructive way to describe the set of $T$-palindromic pencils with bounded rank. 
Theorem 3.1. (Rank-1 decomposition for $T$-palindromic pencils). If $E(\lambda)$ is a $\top$-palindromic $n \times n$ matrix pencil with $\operatorname{rank} E=r \leq n$, then it can be written as

$$
E(\lambda)=\left\{\begin{array}{cc}
v_{1} w_{1}^{\top}+\cdots+v_{r / 2} w_{r / 2}^{\top} & \\
\quad+\left(\operatorname{rev} w_{1}\right) v_{1}^{\top}+\cdots+\left(\operatorname{rev} w_{r / 2}\right) v_{r / 2}^{\top} & \text { if } r \text { is even }, \\
(1+\lambda) u u^{\top}+v_{1} w_{1}^{\top}+\cdots+v_{(r-1) / 2} w_{(r-1) / 2}^{\top} & \\
+\left(\operatorname{rev} w_{1}\right) v_{1}^{\top}+\cdots+\left(\operatorname{rev} w_{(r-1) / 2}\right) v_{(r-1) / 2}^{\top} & \text { if } r \text { is odd },
\end{array}\right.
$$

where $u, v_{1}, \ldots, v_{\lfloor r / 2\rfloor} \in \mathbb{C}^{n}$ and $w_{1}, \ldots, w_{\lfloor r / 2\rfloor} \in \mathbb{C}[\lambda]^{n}$ with $\operatorname{deg} w_{i} \leq 1$, for $i=$ $1, \ldots,\lfloor r / 2\rfloor$.

Proof. Let us asume that the result is true for any $\top$-palindromic pencil being in T-palindromic canonical form as in Theorem 2.1. Let $E(\lambda)$ be an arbitrary $T$ palindromic pencil. By Theorem 2.1, there is some invertible matrix $P$ such that $P E(\lambda) P^{\top}=K_{E}(\lambda)$, with $K_{E}(\lambda)$ being in $\top$-palindromic canonical form. Then $K_{E}(\lambda)$ is of the form (3.1). Now, by setting

$$
\widetilde{v}_{i}=P^{-1} v_{i}, \quad \widetilde{w}_{i}=P^{-1} w_{i}, \quad \widetilde{u}=P^{-1} u,
$$

with $v_{i}, w_{i}, u$ as in (3.1), we arrive at a decomposition like (3.1) for $E(\lambda)$, with $\widetilde{v}_{i}, \widetilde{w}_{i}$, and $\tilde{u}$ instead of $v_{i}, w_{i}, u$, respectively (note that, since $P^{-1}$ is invertible, $\operatorname{rev}\left(P^{-1} v\right)=$ $P^{-1} \operatorname{rev} v$ and $\operatorname{deg}\left(P^{-1} v\right)=\operatorname{deg} v$, for any $\left.v \in \mathbb{C}[\lambda]^{n}\right)$.

Therefore, we may assume that $E(\lambda)$ is given in $\top$-palindromic canonical form. Then, it is a direct sum of blocks of types (i)-(vi) in Theorem 2.1. We are going to show that each of these blocks can be decomposed as a sum of rank-1 pencils in such a way that the whole direct sum is of the form (3.1). Let us show such a decomposition for each type of canonical blocks.

- A block of type (i) can be written as

$$
\begin{aligned}
M_{\alpha}^{\sharp}(\lambda)= & e_{1}\left(\lambda e_{\alpha+1}+e_{\alpha+2}\right)^{\top}+\cdots+e_{\alpha}\left(\lambda e_{2 \alpha}+e_{2 \alpha+1}\right)^{\top} \\
& +\left(e_{\alpha+1}+\lambda e_{\alpha+1}\right) e_{1}^{\top}+\cdots+\left(e_{2 \alpha}+\lambda e_{2 \alpha+1}\right) e_{\alpha}^{\top} .
\end{aligned}
$$

- A block of type (iv) like (2.1) can be written as

$$
\begin{gathered}
e_{1}\left(\left(\lambda+\lambda_{0}\right) e_{\delta+1}+e_{\delta+2}\right)^{\top}+\cdots+e_{\delta-1}\left(\left(\lambda+\lambda_{0}\right) e_{2 \delta-1}+e_{2 \delta}\right)^{\top} \\
\left.+e_{\delta}\left(\left(\lambda+\lambda_{0}\right) e_{2 \delta}\right)^{\top}+\left(1+\lambda \lambda_{0}\right) e_{\delta+1}+\lambda e_{\delta+2}\right) e_{1}^{\top}+\cdots \\
+\left(\left(1+\lambda \lambda_{0}\right) e_{2 \delta+1}+\lambda e_{2 \delta}\right) e_{\delta-1}^{\top}+\left(\left(1+\lambda \lambda_{0}\right) e_{2 \delta}\right) e_{\delta}^{\top} .
\end{gathered}
$$

- A block of type (v) like (2.2) can be decomposed as

$$
\begin{gathered}
e_{2 \varepsilon}\left((1-\lambda) e_{1}+\lambda e_{2}\right)^{\top}+\cdots+e_{\varepsilon+2}\left((1-\lambda) e_{\varepsilon-1}+\lambda e_{\varepsilon}\right)^{\top} \\
+e_{\varepsilon+1}\left((1-\lambda) e_{\varepsilon}+\lambda e_{\varepsilon+1}\right)^{\top} \\
+\left((\lambda-1) e_{1}+e_{2}\right) e_{2 \varepsilon}^{\top}+\cdots+\left((\lambda-1) e_{\varepsilon-1}+e_{\varepsilon}\right) e_{\varepsilon+2}^{\top} \\
+\left((\lambda-1) e_{\varepsilon}+e_{\varepsilon+1}\right) e_{\varepsilon+1}^{\top} .
\end{gathered}
$$

- A block of type (vi) like (2.3) can be decomposed as

$$
\begin{gathered}
e_{2 \eta+1}\left((1+\lambda) e_{1}+\lambda e_{2}\right)^{\top}+\cdots+e_{\eta+2}\left((1+\lambda) e_{\eta}+\lambda e_{\eta+1}\right)^{\top} \\
+\left((\lambda+1) e_{1}+e_{2}\right) e_{2 \eta+1}^{\top}+\cdots+\left((\lambda+1) e_{\eta}+e_{\eta+1}\right) e_{\eta+2}^{\top} \\
+(\lambda+1) e_{\eta+1} e_{\eta+1}^{\top}
\end{gathered}
$$

- For blocks of types (ii) and (iii) the decomposition is similar to the one for blocks of type (iv), replacing $\lambda_{0}= \pm 1$ and $\delta$ by either $\beta$ or $\gamma$. 
Now, joining up in a direct sum all blocks in the $T$-palindromic canonical form of $E(\lambda)$, and padding up with zeroes in all canonical vectors, for each block in the previous rank-1 decompositions, in all positions corresponding to the remaining blocks, we end up with a rank-1 decomposition of the form

$$
v_{1} w_{1}^{\top}+\cdots+v_{s} w_{s}^{\top}+\left(\operatorname{rev} w_{1}\right) v_{1}^{\top}+\cdots+\left(\operatorname{rev} w_{s}\right) v_{s}^{\top}+(1+\lambda)\left(u_{1} u_{1}^{\top}+\cdots+u_{t} u_{t}^{\top}\right),
$$

with $2 s+t=r$, since the rank of $E(\lambda)$ is the sum of the ranks of all canonical blocks. The summands of the form $(1+\lambda) u_{i} u_{i}^{\top}$ in the previos decomposition come from blocks of type (vi). Given a pair of vectors $u, \widetilde{u} \in \mathbb{C}^{n}$, we can write

$$
(1+\lambda)\left(u u^{\top}+\widetilde{u} \widetilde{u}^{\top}\right)=v w^{\top}+(\operatorname{rev} w) v^{\top},
$$

with $v=u+\mathfrak{i} \widetilde{u}, w=\frac{1+\lambda}{2}(u-\mathfrak{i} \widetilde{u})$ (where $\mathfrak{i}$ denotes the imaginary unit). Therefore, if $r$ is even, we can gather these summands in couples to get a decomposition like that in the first expression of (3.1). However, when $r$ is odd, one of the summands remains unpaired, and we arrive at the second expression in (3.1). This proves the result.

The following result, which is closely connected to Theorem 3.1, deals with the set of $n \times n$ matrices that can be decomposed as a sum of rank- 1 matrices in a specific way. It will be key in computing the dimension of the set of $T$-palindromic pencils with bounded rank, which will be key in turn in the proof of Theorem 4.1.

Proposition 3.2. Let $s, n$ be two integers with $0<s \leq n$. Let us define the following sets of $n \times n$ matrices with complex entries:

$$
\begin{gathered}
\mathcal{M}_{s}=\left\{u_{1} v_{1}^{\top}+\cdots+u_{s} v_{s}^{\top}+w_{1} u_{1}^{\top}+\cdots+w_{s} u_{s}^{\top}: \begin{array}{c}
u_{i}, v_{i}, w_{i} \in \mathbb{C}^{n}, \\
\text { for } i=1, \ldots, s
\end{array}\right\} \text { and } \\
\mathcal{N}_{s}=\left\{u u^{\top}+u_{1} v_{1}^{\top}+\cdots+u_{s} v_{s}^{\top}+w_{1} u_{1}^{\top}+\cdots+w_{s} u_{s}^{\top}: \begin{array}{c}
u, u_{i}, v_{i}, w_{i} \in \mathbb{C}^{n}, \\
\text { for } i=1, \ldots, s
\end{array}\right\} .
\end{gathered}
$$

If $\overline{\mathcal{M}}_{s}$ and $\overline{\mathcal{N}}_{s}$ denote the closure of $\mathcal{M}_{s}$ and $\mathcal{N}_{s}$ in the Zariski topology, then

(a) $\operatorname{dim} \mathcal{M}_{s}:=\operatorname{dim} \overline{\mathcal{M}}_{s} \leq s(3 n-2 s)$,

(b) $\operatorname{dim} \mathcal{N}_{s}:=\operatorname{dim} \overline{\mathcal{N}}_{s} \leq s(3 n-2 s-1)+n$.

Proof. Let us define the maps

$$
\begin{aligned}
& \Phi_{1}: \\
& \mathbb{C}^{s n} \times \mathbb{C}^{s n} \times \mathbb{C}^{s n} \\
& \left(u_{1}, \ldots, u_{s} ; v_{1}, \ldots, v_{s} ; w_{1}, \ldots, w_{s}\right) \mapsto \\
& \begin{array}{c}
\mathbb{C}^{n \times n} \\
u_{1} v_{1}^{\top}+\cdots+u_{s} v_{s}^{\top} \\
+w_{1} u_{1}^{\top}+\cdots+w_{s} u_{s}^{\top},
\end{array}
\end{aligned}
$$

and

$$
\begin{array}{ccc}
\Phi_{2}: & \mathbb{C}^{n} \times \mathbb{C}^{s n} \times \mathbb{C}^{s n} \times \mathbb{C}^{s n} & \mathbb{C}^{n \times n} \\
\left(u ; u_{1}, \ldots, u_{s} ; v_{1}, \ldots, v_{s} ; w_{1}, \ldots, w_{s}\right) & \mapsto & u u^{\top}+u_{1} v_{1}^{\top}+\cdots+u_{s} v_{s}^{\top} \\
& & +w_{1} u_{1}^{\top}+\cdots+w_{s} u_{s}^{\top} .
\end{array}
$$

The sets $\mathcal{M}_{s}$ and $\mathcal{N}_{s}$ are the images of, respectively, $\Phi_{1}$ and $\Phi_{2}$, and these images are constructible sets (see, for instance, [28, p. 366]). In particular, they are open dense subsets in their (Zariski) closure. Since $\overline{\mathcal{M}}_{s}$ and $\overline{\mathcal{N}}_{s}$ are algebraic sets, their dimension is the dimension of the tangent space at a general point (namely, in an open dense subset). Then, the dimension of $\overline{\mathcal{M}}_{s}$ and $\overline{\mathcal{N}}_{s}$ is determined by the dimension of the tangent space at a general point of $\mathcal{M}_{s}$ and $\mathcal{N}_{s}$, respectively. In particular, we identify $\operatorname{dim} \mathcal{M}_{s}:=\operatorname{dim} \overline{\mathcal{M}}_{s}$ and $\operatorname{dim} \mathcal{N}_{s}:=\operatorname{dim} \overline{\mathcal{N}}_{s}$. 
Now, we look at the tangent space of each $\mathcal{M}_{s}$ and $\mathcal{N}_{\int}$ at a general point. Let us first consider the set $\mathcal{M}_{s}$. The tangent space is spanned by the $3 s n$ vectors obtained by taking partial derivatives in $\Phi_{1}$, namely the following $3 s n$ matrices with size $n \times n$ :

$$
\begin{array}{cl}
e_{j} v_{i}^{\top}+w_{i} e_{j}^{\top}, & i=1, \ldots, s, j=1, \ldots, n, \\
u_{i} e_{j}^{\top}, & i=1, \ldots, s, j=1, \ldots, n, \\
e_{j} u_{i}^{\top}, & i=1, \ldots, s, j=1, \ldots, n .
\end{array}
$$

Let us write the matrices in (3.4) as vectors in $\mathbb{C}^{n^{2}}$ using the vec operator $[20$, Def. 4.2.9], so that they become

$$
\begin{array}{cl}
v_{i} \otimes e_{j}+e_{j} \otimes w_{i}, & i=1, \ldots s, j=1, \ldots, n, \\
e_{j} \otimes u_{i}, & i=1, \ldots, s, j=1, \ldots, n, \\
u_{i} \otimes e_{j}, & i=1, \ldots, s, j=1, \ldots, n
\end{array}
$$

where $\otimes$ denotes the Kronecker product [20, Def. 4.2.1]. We are going to see that the set of vectors (3.4) contains at least $2 s^{2}$ linearly dependent vectors. A null linear combination of the vectors in $(3.5)$ can be written as

$$
\sum_{i, j}\left[x_{j i}\left(v_{i} \otimes e_{j}+e_{j} \otimes w_{i}\right)+y_{j i}\left(e_{j} \otimes u_{i}\right)+z_{j i}\left(u_{i} \otimes e_{j}\right)\right]=M\left[\begin{array}{c}
\operatorname{vec}(X) \\
\operatorname{vec}(Y) \\
\operatorname{vec}(Z)
\end{array}\right]=0,
$$

with $X=\left[x_{i j}\right], Y=\left[y_{i j}\right], Z=\left[z_{i j}\right]$, for $1 \leq i \leq s, 1 \leq j \leq n$, and $M=$ $\left[M_{1}\left|M_{2}\right| M_{3}\right]$, where $M_{1}$ contains all columns of the form $v_{i} \otimes e_{j}+e_{j} \otimes w_{i}$, $M_{2}$ contains all columns of the form $e_{j} \otimes u_{i}$, and $M_{3}$ contains all columns of the form $u_{i} \otimes e_{j}$ in the left-hand side of (3.6), and they all are ordered in the lexicographic order of the pairs $(i, j)$.

The left-hand side of (3.6) can be written as

$$
\sum_{i=1}^{s}\left[v_{i} \otimes\left(\sum_{j=1}^{n} x_{j i} e_{j}\right)+\left(\sum_{j=1}^{n} x_{j i} e_{j}\right) \otimes w_{i}+\left(\sum_{j=1}^{s} y_{j i} e_{j}\right) \otimes u_{i}+u_{i} \otimes\left(\sum_{j=1}^{n} z_{j i} e_{j}\right)\right] .
$$

If we denote the columns of $X, Y$, and $Z$, respectively, by

$$
\begin{array}{ll}
\bar{x}_{i}:=\operatorname{Col}_{i} X=\sum_{j=1}^{n} x_{j i} e_{j}, & i=1, \ldots, s, \\
\bar{y}_{i}:=\operatorname{Col}_{i} Y=\sum_{j=1}^{n} y_{j i} e_{j}, & i=1, \ldots, s, \\
\bar{z}_{i}:=\operatorname{Col}_{i} Z=\sum_{j=1}^{n} z_{j i} e_{j}, & i=1, \ldots, s,
\end{array}
$$

then (3.6) can be written as

$$
\sum_{i=1}^{s}\left[v_{i} \otimes \bar{x}_{i}+\bar{x}_{i} \otimes w_{i}+\bar{y}_{i} \otimes u_{i}+u_{i} \otimes \bar{z}_{i}\right]=0
$$

Now, we can construct $2 s^{2}$ different solutions to $(3.6)$ as follows. Given a pair $\left(i_{0}, j_{0}\right)$ with $1 \leq i_{0}, j_{0} \leq s$, we set

$$
\begin{aligned}
& s_{1}\left(i_{0}, j_{0}\right)=e_{s+i_{0}} \otimes u_{j_{0}}-e_{2 s+j_{0}} \otimes u_{i_{0}} \quad \text { and } \\
& s_{2}\left(i_{0}, j_{0}\right)=e_{i_{0}} \otimes u_{j_{0}}-e_{s+j_{0}} \otimes v_{i_{0}}-e_{2 s+j_{0}} \otimes w_{i_{0}},
\end{aligned}
$$

where the canonical vectors in $(3.8)$ belong to $\mathbb{C}^{3 s}$. It is straightforward to check that, for a fixed pair $\left(i_{0}, j_{0}\right)$ with $1 \leq i_{0}, j_{0} \leq s$, the vector $s_{1}\left(i_{0}, j_{0}\right)$ corresponds to replacing 
$\bar{y}_{i_{0}}=u_{j_{0}}, \bar{z}_{j_{0}}=-u_{i_{0}}$, and the remaining vectors $\bar{x}_{i}=\bar{y}_{i}=\bar{z}_{i}=0$ in (3.7). Similarly, the vector $s_{2}\left(i_{0}, j_{0}\right)$ corresponds to replacing $\bar{x}_{i_{0}}=u_{j_{0}}, \bar{y}_{j_{0}}=-v_{i_{0}}, \bar{z}_{j_{0}}=-w_{i_{0}}$, and the remaining vectors $\bar{x}_{i}=\bar{y}_{i}=\bar{z}_{i}=0$ in (3.7). Then, $s_{1}\left(i_{0}, j_{0}\right)$ and $s_{2}\left(i_{0}, j_{0}\right)$ give $2 s^{2}$ different solutions of (3.6) for general vectors $u_{i}, v_{i}, w_{i}$. Now, let us prove that, for general vectors $u_{i}, v_{i}, w_{i}$, these solutions are linearly independent.

Let us asume that there is a null linear combination of the solutions

$$
\sum_{i, j=1}^{s} \alpha_{i j} s_{1}(i, j)+\sum_{i, j=1}^{s} \beta_{i j} s_{2}(i, j)=0
$$

with $\alpha_{i j}, \beta_{i j} \in \mathbb{C}$, where we have replaced $\left(i_{0}, j_{0}\right)$ by $(i, j)$ for simplicity. Then, replacing (3.8) into this expression, we arrive at

$$
\sum_{i, j=1}^{s} \alpha_{i j}\left(e_{s+i} \otimes u_{j}-e_{2 s+j} \otimes u_{i}\right)+\sum_{i, j=1}^{s} \beta_{i j}\left(e_{i} \otimes u_{j}-e_{s+j} \otimes v_{i}-e_{2 s+j} \otimes w_{i}\right)=0
$$

Looking at the summands in the left-hand side of (3.10) whose first vector in the Kronecker product is of the form $e_{k}$, for $1 \leq k \leq s$, and equating to zero, we arrive at

$$
\begin{array}{cc}
\beta_{11} u_{1}+\cdots+\beta_{1 s} u_{s} & =0 \\
\vdots & \vdots \\
\beta_{s 1} u_{1}+\cdots+\beta_{s s} u_{s} & =0 .
\end{array}
$$

For a general point in $\mathrm{Pal}_{r}$, the set $\left\{u_{1}, \ldots, u_{s}\right\}$ is linearly independent, since $s \leq n$, by hypothesis. Therefore, (3.11) implies that $\beta_{i j}=0$, for all $1 \leq i, j \leq s$. Now, looking at the summands in (3.10) whose first vector in the Kronecker product is of the form $e_{k}$, with $s+1 \leq k \leq 2 s$, and equating to zero, we arrive at

$$
\begin{aligned}
& \alpha_{11} u_{1}+\cdots+\alpha_{1 s} u_{s}=0, \\
& \alpha_{s 1} u_{1}+\cdots+\alpha_{s s} u_{s}=\dot{0} \text {. }
\end{aligned}
$$

Again, the set $\left\{u_{1}, \ldots, u_{s}\right\}$ is linearly independent for a general point in $\mathrm{Pal}_{r}$, so (3.12) implies that $\alpha_{i j}=0$, for all $1 \leq i, j \leq s$. Therefore, the only null linear combination (3.9) for a general point in $\mathrm{Pal}_{r}$ is the one with $\alpha_{i j}=\beta_{i j}=0$, for all $1 \leq i, j \leq s$, which implies that $s_{1}(i, j)$ and $s_{2}(i, j)$, for $1 \leq i, j \leq s$, are linearly independent, as wanted. As a consequence, $\operatorname{dim} \mathcal{M}_{s} \leq 3 n s-2 s^{2}=s(3 n-2 s)$, as claimed.

Now, let us address the proof for the set $\mathcal{N}_{f}$. The tangent space at a general point is the linear space spanned by the following $3 s n+n$ vectors, obtained from the partial derivatives of $\Phi_{2}$ :

$$
\begin{array}{cc}
e_{j} v_{i}^{\top}+w_{i} e_{j}^{\top}, & i=1, \ldots, s, j=1, \ldots, n, \\
u_{i} e_{j}^{\top}, & i=1, \ldots, s, j=1, \ldots, n, \\
e_{j} u_{i}^{\top}, & i=1, \ldots, s, j=1, \ldots, n, \\
e_{j} u^{\top}+u e_{j}^{\top}, & j=1, \ldots, n,
\end{array}
$$

and, applying again the vec operator, these vectors become

$$
\begin{array}{cc}
v_{i} \otimes e_{j}+e_{j} \otimes w_{i}, & i=1, \ldots, s, j=1, \ldots, n, \\
e_{j} \otimes u_{i}, & i=1, \ldots, s, j=1, \ldots, n, \\
u_{i} \otimes e_{j}, & i=1, \ldots, s, j=1, \ldots, n, \\
u \otimes e_{j}+e_{j} \otimes u, & j=1, \ldots, n .
\end{array}
$$


A null linear combination of the vectors in (3.13) is of the form

$$
\begin{gathered}
\sum_{i, j}\left[x_{j i}\left(v_{i} \otimes e_{j}+e_{j} \otimes w_{i}\right)+y_{j i}\left(e_{j} \otimes u_{i}\right)+z_{j i}\left(u_{i} \otimes e_{j}\right)+t_{j}\left(u \otimes e_{j}+e_{j} \otimes u\right)\right] \\
=\widetilde{M}\left[\begin{array}{c}
\operatorname{vec}(X) \\
\operatorname{vec}(Y) \\
\operatorname{vec}(Z) \\
\operatorname{vec}(T)
\end{array}\right]=0,
\end{gathered}
$$

with $X=\left[x_{i j}\right], Y=\left[y_{i j}\right], Z=\left[z_{i j}\right], T=\left[t_{i}\right]$, for $1 \leq i \leq s, 1 \leq j \leq n$, and $M=$ $\left[M_{1}\left|M_{2}\right| M_{3} \mid M_{4}\right]$, where $M_{1}$ contains all columns of the form $v_{i} \otimes e_{j}+e_{j} \otimes w_{i}$, $M_{2}$ contains all columns of the form $e_{j} \otimes u_{i}, M_{3}$ contains all columns of the form $u_{i} \otimes e_{j}$, and $M_{4}$ contains all columns of the form $u \otimes e_{i}+e_{i} \otimes u$ in the left-hand side of (3.14), and they all are ordered in the lexicographic order of the pairs $(i, j)$. With the same notation $\bar{x}_{i}, \bar{y}_{i}, \bar{z}_{i}$ for the columns of $X, Y, Z$ as before, together with $\bar{t}=\sum_{j=1}^{n} t_{j} e_{j},(3.14)$ is equivalent to

$$
\sum_{i=1}^{s}\left[v_{i} \otimes \bar{x}_{i}+\bar{x}_{i} \otimes w_{i}+\bar{y}_{i} \otimes u_{i}+u_{i} \otimes \bar{z}_{i}\right]+u \otimes \bar{t}+\bar{t} \otimes u=0 .
$$

Now, for each $\left(i_{0}, j_{0}\right)$, with $1 \leq i_{0}, j_{0} \leq s$, we define $s_{1}\left(i_{0}, j_{0}\right)$ and $s_{2}\left(i_{0}, j_{0}\right)$ as in $(3.8)$, with the only difference that now the canonical vectors belong to $\mathbb{C}^{3 s+1}$. We also set, for each $1 \leq i_{0} \leq s$,

$$
s_{3}\left(i_{0}\right)=-e_{s+i_{0}} \otimes u_{i_{0}}-e_{2 s+i_{0}} \otimes u_{i_{0}}+e_{3 s+1} \otimes u_{i_{0}},
$$

where, again, the canonical vectors in (3.16) belong to $\mathbb{C}^{3 s+1}$. The vectors (3.8) are solutions of (3.15), for the same reason as in the preceding case. The $n$ vectors $s_{3}(i)$ in (3.16) correspond to replacing $\bar{y}_{i_{0}}=\bar{z}_{i_{0}}=-u_{i_{0}}, \bar{t}=u_{i_{0}}$, and $\bar{x}_{i}, \bar{y}_{i}, \bar{z}_{i}=0$, for $i \neq i_{0}$ in (3.14). Then, $s_{3}(i)$ is also a solution of (3.15), for $1 \leq i \leq s$. It remains to prove that $s_{1}(i, j), s_{2}(i, j)$, and $s_{3}(i)$ are linearly independent for a general vector in $\mathrm{Pal}_{r}$. This follows similar arguments to the ones for $\mathcal{M}_{s}$. In particular, if

$$
\sum_{i, j=1}^{s} \alpha_{i j} s_{1}(i, j)+\sum_{i, j=1}^{s} \beta_{i j} s_{2}(i, j)+\sum_{i=1}^{s} \gamma_{i} s_{3}(i)=0
$$

is a null linear combination of $s_{1}(i, j), s_{2}(i, j)$, and $s_{3}(i)$, then replacing (3.8) and (3.16) in (3.17) we arrive at

$$
\begin{gathered}
\sum_{i, j=1}^{s} \alpha_{i j}\left(e_{s+i} \otimes u_{i}-e_{2 s+j} \otimes u_{j}\right)+\sum_{i, j=1}^{s} \beta_{i j}\left(e_{i} \otimes u_{j}-e_{s+j} \otimes v_{i}-e_{2 s+j} \otimes w_{i}\right) \\
+\sum_{i=1}^{s} \gamma_{i}\left(-e_{s+i} \otimes u_{i}-e_{2 s+i} \otimes u_{i}+e_{3 s+1} \otimes u_{i}\right)=0 .
\end{gathered}
$$

Looking at the summands whose first term in the Kronecker product is of the form $e_{3 s+1}$ we arrive at $\gamma_{1} u_{1}+\cdots+\gamma_{s} u_{s}=0$, and this implies, provided that $\left\{u_{1}, \ldots, u_{s}\right\}$ is linearly independent, that $\gamma_{1}=\cdots=\gamma_{s}=0$. Then, looking again at the terms whose first vector in the Kronecker product is of the form $e_{k}$, with $1 \leq k \leq s$, we conclude 
that, as long as $\left\{u_{1}, \ldots, u_{s}\right\}$ is linearly independent, $\beta_{i j}=0$, for all $1 \leq i, j \leq s$. Finally, looking at the terms whose first vector in the Kronecker product is of the form $e_{k}$, with $s+1 \leq k \leq 2 s$, we get $\alpha_{i j}=0$, for all $1 \leq i, j \leq s$. This implies that the $2 s^{2}+s$ solutions $s_{1}(i, j), s_{2}(i, j)$, and $s_{3}(j)$ are linearly independent, so $\operatorname{dim} \mathcal{N}_{s} \leq 3 s n+n-2 s^{2}-s=s(3 n-2 s-1)+n$, as wanted.

4. Main results. The main results in sections 4.1 and 4.2 are the analogues of those in the recent paper [12], for skew-symmetric matrix pencils. The proof for the skew-symmetric structure in that paper is based on the fact that a given skewsymmetric pencil $S_{1}(\lambda)$ is in the closure of the congruence orbit of another skewsymmetric pencil $S_{2}(\lambda)$ if and only if $S_{1}(\lambda)$ is in the closure of the strict equivalence orbit of $S_{2}(\lambda)$. In other words, if there is a sequence of pencils strictly equivalent to $S_{2}(\lambda)$ which converges to $S_{1}(\lambda)$, then there is also a sequence of pencils which are congruent to $S_{2}(\lambda)$ and that converges to $S_{1}(\lambda)$. This is a very strong result from [13], and it is not yet known whether an analogous result is true or not for $T$-palindromic or $T$-alternating structures. Therefore, a relevant part of the proof of Theorem 4.1, which is the main result in section 4.1 , follows a completely different technique compared to the ones in [12], relying on Proposition 3.2.

We analyze separately the following structures: (i) $\top$-palindromic and $\top$-antipalindromic structures (section 4.1), and (ii) T-alternating structures (section 4.2). The $T$-palindromic and $T$-antipalindromic structures are related to each other by the elementary change of variables $\lambda \mapsto-\lambda$, so the results for one of these structures are directly extended to the other one. Similarly, the $T$-even and $T$-odd structures are related by reversing the order of the coefficients $A_{0}$ and $A_{1}$, so it is again enough to analyze just one of them. The $T$-palindromic and the $T$-alternating structures are also related by particular cases of Möbus transformations (know as Cayley transformations). Using these transformations, the results for $\top$-palindromic pencils can be easily translated to $T$-alternating pencils as well.

4.1. $T$-palindromic and $T$-antipalindromic pencils. Our main results in this section show that the sets of $T$-palindromic and $\top$-antipalindromic matrix pencils with bounded (defficient) rank are irreducible, and provide the dimension of these sets. They also provide the generic canonical form of these pencils. We start with the T-palindromic structure.

TheOREM 4.1. (The set of T-palindromic pencils with bounded rank). Let $r$ be an integer with $0 \leq r<n$. The set $\mathrm{Pal}_{r}$ is an irreducible algebraic set with dimension

$$
\operatorname{dim} \mathrm{Pal}_{r}= \begin{cases}\frac{r}{2} \cdot(3 n-r) & \text { if } r \text { is even } \\ \frac{r-1}{2} \cdot(3 n-r)+n & \text { if } r \text { is odd }\end{cases}
$$

Moreover, if $r$ is even, then $\mathrm{Pal}_{r}$ is the closure of the congruence orbit of the pencil

$$
K_{P}^{e}(\lambda):=\operatorname{diag}(\overbrace{M_{\alpha+1}^{\sharp}(\lambda), \ldots, M_{\alpha+1}^{\sharp}(\lambda)}^{s}, \overbrace{M_{\alpha}^{\sharp}(\lambda), \ldots, M_{\alpha}^{\sharp}}^{n-r-s}(\lambda)),
$$

where $r / 2=(n-r) \alpha+s$ is the Euclidean division of $r / 2$ by $n-r$. If $r$ is odd, then $\mathrm{Pal}_{r}$ is the closure of the congruence orbit of

$$
K_{P}^{o}(\lambda):=\operatorname{diag}\left(1+\lambda, K_{P}^{e}(\lambda)\right),
$$

with $K_{P}^{e}(\lambda)$ as in $(4.1)$, but now $(r-1) / 2=(n-r) \alpha+s$ is the Euclidean division of $(r-1) / 2$ by $n-r$. 
Proof. Let us first consider the case $r$ even. The codimension of the orbit of $K_{P}^{e}(\lambda)$ in (4.1) can be computed using the formula in [6, Thm. 2] and the relationship between $\operatorname{KCF}\left(A+\lambda A^{\top}\right)$ and the CFC of $A$ provided in [3, Thm. 4] (see the paragraph right after Theorem 2.1). In particular, if $K_{P}^{e}(\lambda)=A+\lambda A^{\top}$, then

$$
\operatorname{CFC}(A)=\operatorname{diag}(\underbrace{J_{2 \alpha+3}(0), \ldots, J_{2 \alpha+3}(0)}_{s}, \underbrace{J_{2 \alpha+1}(0), \ldots, J_{2 \alpha+1}(0)}_{n-r-s}) .
$$

Now, applying [6, Thm. 2], the codimension of $K_{P}^{e}(\lambda)$ is $c_{K_{P}^{e}}=c_{0}+c_{00}$. The quantity $c_{0}$ is the "codimension" of individual blocks $J_{k}(0)$, and is obtained by adding up $\lceil k / 2\rceil$, for each block $J_{k}(0)$, whereas $c_{00}$ is due to the "interactions" between two different blocks, taking each pair $\left(J_{k}(0), J_{\ell}(0)\right)$ with $k \leq \ell$ only once, and is equal to (a) $k$ if $k$ is even, (b) $\ell$ if $k$ is odd and $k \neq \ell$, and (c) $k+1$ if $k$ is odd and $k=\ell$. In particular, for the blocks in $\mathrm{CFC}(A)$ above,

$$
c_{0}=\sum_{i=1}^{s}\left\lceil\frac{2 \alpha+3}{2}\right\rceil+\sum_{i=1}^{n-r-s}\left\lceil\frac{2 \alpha+1}{2}\right\rceil=s(\alpha+2)+(n-r-s)(\alpha+1)=n-\frac{r}{2}
$$

and

$$
c_{00}=\left(\begin{array}{l}
s \\
2
\end{array}\right)(2 \alpha+4)+\left(\begin{array}{c}
n-r-s \\
2
\end{array}\right)(2 \alpha+2)+s(n-r-s)(2 \alpha+1) .
$$

After some manipulations in (4.4) we arrive at

$$
c_{00}=(n-r-1)\left(n-\frac{r}{2}\right) \text {. }
$$

Now, adding up, we get $c_{K_{P}^{e}}=c_{0}+c_{00}=(n-r / 2)(n-r)$. Then, the dimension of the congruence orbit of $K_{P}^{e}(\lambda)$ is

$$
\operatorname{dim} \mathcal{O}_{c}\left(K_{P}^{e}\right)=n^{2}-c_{K_{P}^{e}}=\frac{r}{2}(3 n-r)
$$

Now, since $\mathcal{O}_{c}\left(K_{P}^{e}\right) \subseteq \mathrm{Pal}_{r}$ and $\mathrm{Pal}_{r}$ is an algebraic (hence closed) set, it follows that $\overline{\mathcal{O}}_{c}\left(K_{P}^{e}\right) \subseteq \mathrm{Pal}_{r}$. In order to prove that the inclusion is an identity, it suffices to see that the dimension of $\mathrm{Pal}_{r}$ is, at most, $\frac{r}{2}(3 n-r)$, and that $\mathrm{Pal}_{r}$ is irreducible.

By decomposing $w_{i}=w_{i 0}+\lambda w_{i 1}$ in $(3.1)$, for $i=1, \ldots, r / 2$, any $T$-palindromic pencil $L(\lambda)$ with rank at most $r$ can be written as

$$
\begin{aligned}
L(\lambda)= & v_{1} w_{10}^{\top}+\cdots+v_{r / 2} w_{r / 2,0}^{\top}+w_{11} v_{1}^{\top}+\cdots+w_{r / 2,1} v_{r / 2}^{\top} \\
& +\lambda\left(w_{10} v_{1}^{\top}+\cdots+w_{r / 2,0} v_{r / 2}^{\top}+v_{1} w_{11}^{\top}+\cdots+v_{r / 2} w_{r / 2,1}^{\top}\right) .
\end{aligned}
$$

Then, the pencil $L(\lambda)$ is uniquely determined by its trailing coefficient. In other words, the set $\mathrm{Pal}_{r}$ is in one-to-one correspondence with the set of matrices that can be written in the form $u_{1} v_{1}^{\top}+\cdots+u_{r / 2} v_{r / 2}^{\top}+w_{1} u_{1}^{\top}+\cdots+w_{r / 2} u_{r / 2}^{\top}$. Proposition 3.2 (a) with $s=r / 2$ guarantees that the dimension of this set is at most $\frac{r}{2}(3 n-r)$.

To prove that $\mathrm{Pal}_{r}$ is irreducible we proceed as follows. $\mathrm{Pal}_{r}$ is the image of the following (polynomial) map:

$$
\begin{array}{cccc}
\Phi: & \mathbb{C}^{\frac{3 r n}{2}} & \mathbb{C}^{n \times n} \times \mathbb{C}^{n \times n} \\
\left(v_{1}, \ldots, v_{r / 2} ;\right. & & \left(v_{1} w_{10}^{\top}+\cdots+v_{r / 2} w_{r / 2,0}^{\top}+w_{11} v_{1}^{\top}+\cdots+w_{r / 2,1} v_{r / 2}^{\top},\right. \\
w_{10}, \ldots, w_{r / 2,0} ; \\
\left.w_{11}, \ldots, w_{r / 2,1}\right)
\end{array} \quad \mapsto \quad \begin{gathered}
\left.w_{10} v_{1}^{\top}+\cdots+w_{r / 2,0} v_{r / 2}^{\top}+v_{1} w_{11}^{\top}+\cdots+v_{r / 2} w_{r / 2,1}^{\top}\right) .
\end{gathered}
$$


Then, assume $\mathrm{Pal}_{r}=\Phi\left(\mathbb{C}^{\frac{3 r n}{2}}\right)=X \cup Y$, with $X, Y$ being algebraic sets, which is equivalent to $\mathbb{C}^{\frac{3 r n}{2}}=\Phi^{-1}(X) \cup \Phi^{-1}(Y)$. In general, if $\Phi$ is a polynomial map and $Z$ is an algebraic set, then $\Phi^{-1}(Z)$ is an algebraic set as well (to see this, just notice that $\Phi^{-1}(Z)$ is the set of common zeroes of $p_{1} \circ \Phi, \ldots, p_{m} \circ \Phi$, where $Z$ is defined as the set of common zeroes of the multivariable polynomials $\left.p_{1}, \ldots, p_{m}\right)$. Then, both $\Phi^{-1}(X)$ and $\Phi^{-1}(Y)$ are algebraic sets and, since $\mathbb{C}^{\frac{3 r n}{2}}$ is irreducible, this implies that either $\mathbb{C}^{\frac{3 r n}{2}}=\Phi^{-1}(X)$ or $\mathbb{C}^{\frac{3 r n}{2}}=\Phi^{-1}(Y)$, which in turn implies either $\Phi\left(\mathbb{C}^{\frac{3 r n}{2}}\right)=X$ or $\Phi\left(\mathbb{C}^{\frac{3 r n}{2}}\right)=Y$. As a consequence, $\Phi\left(\mathbb{C}^{\frac{3 r n}{2}}\right)=\mathrm{Pal}_{r}$ is irreducible, and the proof for the case $r$ even is complete.

Now, let us consider the case $r$ odd. In this case (see [3, Thm. 4] or the paragraph right after Theorem 2.1),

$$
\mathrm{CFC}(A)=\operatorname{diag}(\underbrace{J_{2 \alpha+3}(0), \ldots, J_{2 \alpha+3}(0)}_{s}, \underbrace{J_{2 \alpha+1}(0), \ldots, J_{2 \alpha+1}(0)}_{n-r-s}, 1) .
$$

Therefore, the codimension of $\mathcal{O}_{c}\left(K_{P}^{o}\right)$ is $c_{K_{P}^{o}}=c_{0}+c_{00}+c_{01}$, with $c_{0}$ and $c_{00}$ as in (4.3) and (4.4), respectively. The term $c_{01}$ is due to the presence of the last block equal to 1 in $\operatorname{CFC}(A)$, which is a so-called type $I$ block [3, Thm. 3]. For a given matrix $A$, the term $c_{01}$ is equal to the product of the number of type 0 blocks in $\mathrm{CFC}(A)$ (that is, blocks of the form $\left.J_{k}(0)\right)$ and the sum of the sizes of all type I blocks in $\operatorname{CFC}(A)$ (see [6, Thm. 2]). In the case of $A$ above, it is equal to $c_{01}=n-r$. Now, however, $c_{0}=n-(r+1) / 2$, and with similar manipulations as for the $r$ even case, we can get $c_{00}=(n-r-1)(n-(r+1) / 2)$. Adding up,

$$
c_{K_{P}^{o}}=c_{0}+c_{00}+c_{01}=(n-r)\left(n-\frac{r-1}{2}\right) \text {. }
$$

Then, the dimension of the congruence orbit of $K_{P}^{o}(\lambda)$ is

$$
\operatorname{dim} \mathcal{O}_{c}\left(K_{P}^{o}\right)=n^{2}-c_{K_{P}^{o}}=\frac{r-1}{2} \cdot(3 n-r)+n .
$$

Again, it remains to prove that the dimension of $\mathrm{Pal}_{r}$ is, at most, $\frac{r-1}{2} \cdot(3 n-r)+n$ and that $\mathrm{Pal}_{r}$ is irreducible. Writing again $w_{i}=w_{i 0}+\lambda w_{i 1}$ in $(3.1)$, for $i=1, \ldots,(r-1) / 2$, any $\top$-palindromic matrix pencil $L(\lambda)$ with rank at most $r$ can be written as

$$
\begin{aligned}
L(\lambda)= & u u^{\top}+v_{10} w_{10}^{\top}+\cdots+v_{r / 2} w_{r / 2,0}^{\top}+w_{11} v_{1}^{\top}+\cdots+w_{r / 2,1} v_{r / 2}^{\top} \\
& +\lambda\left(w_{10} v_{1}^{\top}+\cdots+w_{r / 2,0} v_{r / 2}^{\top}+v_{1} w_{11}^{\top}+\cdots+v_{r / 2} w_{r / 2,1}^{\top}\right) .
\end{aligned}
$$

As before, the pencil $L(\lambda)$ is uniquely determined by its trailing coefficient. In other words, the set $\mathrm{Pal}_{r}$ is in one-to-one correspondence with the set of matrices that can be written in the form $u u^{\top}+u_{1} v_{1}^{\top}+\cdots+u_{(r-1) / 2} v_{(r-1) / 2}^{\top}+w_{1} u_{1}^{\top}+\cdots+w_{(r-1) / 2} u_{(r-1) / 2}^{\top}$. Proposition 3.2 (b) with $s=(r-1) / 2$ guarantees that the dimension of this set is at most $\frac{r-1}{2}(3 n-r)+n$, as wanted.

The proof of the irreducibility of $\mathrm{Pal}_{r}$ in this case follows the same arguments as for the $r$ even case.

Now we state the counterpart of Theorem 4.1 for $T$-antipalindromic pencils.

THEOREM 4.2. (The set of $T$-antipalindromic pencils with bounded rank). Let $r$ be an integer with $0 \leq r<n$. The set $\mathrm{Apal}_{r}$ is an irreducible algebraic set with dimension 


$$
\operatorname{dim} \mathrm{Apal}_{r}= \begin{cases}\frac{r}{2} \cdot(3 n-r) & \text { if } r \text { is even } \\ \frac{r-1}{2} \cdot(3 n-r)+n & \text { if } r \text { is odd } .\end{cases}
$$

Moreover, if $r$ is even, then $\mathrm{Apal}_{r}$ is the closure of the congruence orbit of the pencil

$$
K_{A}^{e}(\lambda):=\operatorname{diag}(\overbrace{M_{\alpha+1}^{-\sharp}(\lambda), \ldots, M_{\alpha+1}^{-\sharp}(\lambda)}^{s}, \overbrace{M_{\alpha}^{-\sharp}(\lambda), \ldots, M_{\alpha}^{-\sharp}}^{n-r-s}(\lambda)),
$$

where $r / 2=(n-r) \alpha+s$ is the Euclidean division of $r / 2$ by $n-r$. If $r$ is odd, then $\mathrm{Apal}_{r}$ is the closure of the congruence orbit of

$$
K_{A}^{o}(\lambda):=\operatorname{diag}\left(1-\lambda, K_{A}^{e}(\lambda)\right),
$$

with $K_{A}^{e}(\lambda)$ as in (4.5), but now $(r-1) / 2=(n-r) \alpha+s$ is the Euclidean division of $(r-1) / 2$ by $n-r$.

Proof. The result is an immediate consequence of Theorem 4.1, since a matrix pencil $P(\lambda)$ is $T$-antipalindromic if and only if $P(-\lambda)$ is $T$-palindromic.

Theorems 4.1 and 4.2 give the generic canonical form of $T$-palindromic and $T$ antipalindromic $n \times n$ pencils with rank at most $r$.

COROLlary 4.3. (Generic canonical form of $T$-palindromic and $T$-antipalindromic pencils with bounded rank). Let $0 \leq r<n$. The generic canonical structure of $n \times n$ $\top$-palindromic (respectively, $T$-antipalindromic) $n \times n$ matrix pencils with rank at most $r$ is (4.1) (resp., (4.5)) if $r$ is even, and (4.2) (resp., (4.6)) if $r$ is odd.

We have seen in the proof of Theorem 4.1 that the set $\mathrm{Pal}_{r}$ can be identified with the set $\mathcal{M}_{r / 2}$ in (3.2) if $r$ is even, or $\mathcal{N}_{(r-1) / 2}$ in (3.3) if $r$ is odd. The only restriction for $r$ here is that $r<n$, which is equivalent to $s<n / 2$. Then Theorem 4.1 allows us to conclude that, provided that $s<n / 2$, the bounds obtained in Proposition 3.2 (a)-(b) are the dimensions of the sets $\mathcal{M}_{s}$ and $\mathcal{N}_{s}$.

Corollary 4.4. If $\mathcal{M}_{s}$ and $\mathcal{N}_{s}$ are as in (3.2) and (3.3), respectively, and $s<$ $n / 2$, then

(a) $\operatorname{dim} \mathcal{M}_{s}=\operatorname{dim} \overline{\mathcal{M}}_{s}=s(3 n-2 s)$ and

(b) $\operatorname{dim} \mathcal{N}_{s}=\operatorname{dim} \overline{\mathcal{N}}_{s}=s(3 n-2 s-1)+n$.

4.2. T-alternating pencils. Here we provide the counterpart of Theorems 4.1 and 4.2 for $T$-alternating pencils.

TheOREM 4.5. (The set of T-even pencils with bounded rank). Let $r$ be an integer with $0 \leq r<n$. The set $\operatorname{Even}_{r}$ is an irreducible algebraic set with dimension

$$
\operatorname{dim} \operatorname{Even}_{r}= \begin{cases}\frac{r}{2} \cdot(3 n-r) & \text { if } r \text { is even }, \\ \frac{r-1}{2} \cdot(3 n-r)+n & \text { if } r \text { is odd } .\end{cases}
$$

Moreover, if $r$ is even, then $\operatorname{Even}_{r}$ is the closure of the congruence orbit of

$$
K_{E}^{e}(\lambda):=\operatorname{diag}(\overbrace{M_{\alpha+1}^{b}(\lambda), \ldots, M_{\alpha+1}^{b}(\lambda)}^{s}, \overbrace{M_{\alpha}^{b}(\lambda), \ldots, M_{\alpha}^{b}(\lambda)}^{n-r-s}),
$$


where $\frac{r}{2}=(n-r) \alpha+s$ is the Euclidean division of $r / 2$ by $n-r$. If $r$ is odd, then Even $_{r}$ is the closure of the congruence orbit of

$$
K_{E}^{o}(\lambda):=\operatorname{diag}\left(1, K_{E}^{e}(\lambda)\right),
$$

with $K_{E}^{e}(\lambda)$ as in (4.7), but now $\frac{r-1}{2}=(n-r) \alpha+s$ is the Euclidean division of $(r-1) / 2$ by $n-r$.

Proof. Let $\mathcal{C}_{+1}$ and $\mathcal{C}_{-1}$ be the Cayley transforms in the set of matrix polynomials defined by

$$
\mathcal{C}_{-1}(Q)(\lambda)=(1+\lambda) Q\left(\frac{\lambda-1}{1+\lambda}\right) \quad \text { and } \quad \mathcal{C}_{+1}(Q)(\lambda)=(1-\lambda) Q\left(\frac{1+\lambda}{1-\lambda}\right)
$$

where $Q(\lambda)$ is any matrix polynomial (see [22]). It is straightforward to see that $\mathcal{C}_{+1}\left(K_{E}^{e}\right)=K_{P}^{e}$ and $\mathcal{C}_{+1}\left(K_{E}^{o}\right)=K_{P}^{o}$, with $K_{P}^{e}$ and $K_{P}^{o}$ being as in (4.1) and (4.2), respectively. Note that for a given pencil $A_{0}+\lambda A_{1}$ we have

$\mathcal{C}_{-1}\left(A_{0}+\lambda A_{1}\right)=A_{0}-A_{1}+\lambda\left(A_{0}+A_{1}\right), \quad \mathcal{C}_{+1}\left(A_{0}+\lambda A_{1}\right)=A_{0}+A_{1}+\lambda\left(A_{1}-A_{0}\right)$.

In particular, $P(\lambda)$ is $T$-palindromic if and only if $\mathcal{C}_{+1}(P)$ is $T$-even (see also [22, Thm. 2.7]). From the definition of $\mathcal{C}_{+1}$ and $\mathcal{C}_{-1}$ is clear that both maps preserve the rank, that is $\operatorname{rank} \mathcal{C}_{+1}\left(A_{0}+\lambda A_{1}\right)=\operatorname{rank} \mathcal{C}_{-1}\left(A_{0}+\lambda A_{1}\right)=\operatorname{rank} A_{0}+\lambda A_{1}$, for any matrix pencil $A_{0}+\lambda A_{1}$. Moreover, $\mathcal{C}_{-1}\left(\mathcal{C}_{+1}\right)\left(A_{0}+\lambda A_{1}\right)=2\left(A_{0}+\lambda A_{1}\right)$. Therefore, $\mathcal{C}_{+1}: \mathrm{Pal}_{\mathrm{r}} \longrightarrow$ Even $_{r}$ is an isomorphism of algebraic sets [30, definition on p. 29]. As a consequence, $\operatorname{dim} \mathrm{Pal}_{r}=\operatorname{dim}_{\mathrm{Even}_{r}}$ [30, Cor. 2, Chap. II, sect. 1.3, p. 88] and Even $_{r}$ is an irreducible algebraic set.

It is also immediate by definition of $\mathcal{C}_{+1}$ that $\mathcal{C}_{+1}\left(\mathcal{O}_{c}\left(K_{E}^{e}\right)\right)=\mathcal{O}_{c}\left(K_{P}^{e}\right)$ and $\mathcal{C}_{+1}\left(\mathcal{O}_{c}\left(K_{E}^{o}\right)\right)=\mathcal{O}_{c}\left(K_{P}^{o}\right)$. Since $\mathcal{C}_{+1}$ is an isomorphism, $\operatorname{dim} \mathcal{C}_{+1}\left(\mathcal{O}_{c}\left(K_{E}^{e}\right)\right)=$ $\operatorname{dim} \mathcal{O}_{c}\left(K_{P}^{e}\right)$ and $\operatorname{dim} \mathcal{C}_{+1}\left(\mathcal{O}_{c}\left(K_{E}^{o}\right)\right)=\operatorname{dim} \mathcal{O}_{c}\left(K_{P}^{o}\right)$. Therefore, Theorem 4.1 implies that the dimensions of $\mathcal{O}_{c}\left(K_{E}^{o}\right)$ and $\mathcal{O}_{c}\left(K_{E}^{e}\right)$ are as claimed in the statement.

Since $\mathcal{O}_{c}\left(K_{E}^{e}\right) \subseteq$ Even $_{r}$ and $\mathcal{O}_{c}\left(K_{E}^{o}\right) \subseteq$ Even $_{r}$, and both $\mathcal{O}_{c}\left(K_{E}^{e}\right)$ and $\mathcal{O}_{c}\left(K_{E}^{e}\right)$ have the same dimension as Even $_{r}$, depending on whether $r$ is even or odd, the result for the $T$-even structure follows.

ThEOREM 4.6. (The set of $T$-odd pencils with bounded rank). Let $r$ be an integer with $0 \leq r<n$. The set $\operatorname{Odd}_{r}$ is an irreducible algebraic set with dimension

$$
\operatorname{dim} \operatorname{Odd}_{r}= \begin{cases}\frac{r}{2} \cdot(3 n-r) & \text { if } r \text { is even, } \\ \frac{r-1}{2} \cdot(3 n-r)+n & \text { if } r \text { is odd. }\end{cases}
$$

Moreover, if $r$ is even, then $\mathrm{Odd}_{r}$ is the closure of the congruence orbit of

$$
K_{O}^{e}(\lambda):=\operatorname{diag}(\overbrace{M_{\alpha+1}^{-b}(\lambda), \ldots, M_{\alpha+1}^{-b}(\lambda)}^{s}, \overbrace{M_{\alpha}^{-b}(\lambda), \ldots, M_{\alpha}^{-b}(\lambda)}^{n-r-s}),
$$

where $\frac{r}{2}=(n-r) \alpha+s$ is the Euclidean division of $r / 2$ by $n-r$. If $r$ is odd, then $\mathrm{Odd}_{r}$ is the closure of the congruence orbit of

$$
K_{O}^{o}(\lambda):=\operatorname{diag}\left(\lambda, K_{O}^{e}(\lambda)\right),
$$

with $K_{O}^{e}(\lambda)$ as in (4.10), but now $\frac{r-1}{2}=(n-r) \alpha+s$ is the Euclidean division of $(r-1) / 2$ by $n-r$. 
Proof. The result is an immediate consequence of Theorem 4.5, since a matrix pencil $A_{0}+\lambda A_{1}$ is $\mathrm{T}$-odd if and only if its reversal $A_{1}+\lambda A_{0}$ is $\mathrm{T}$-even.

As for the previous structures, Theorems 4.5 and 4.6 give the generic canonical form of T-alternating $n \times n$ pencils with rank at most $r$.

Corollary 4.7. (Generic canonical form of $T$-alternating pencils with bounded rank). Let $0 \leq r<n$. The generic canonical structure of $n \times n \top$-even (respectively, $\top$-odd) $n \times n$ matrix pencils with rank at most $r$ is (4.7) (resp., (4.10)) if $r$ is even, and (4.8) (resp., (4.11)) if $r$ is odd.

4.3. Connection with the full-rank and nonstructured cases. The case $n=r$, where the matrix pencils are allowed to be of full rank, deserves some comment. In this case, the generic canonical form for pencils enjoying any of the structures considered in the paper does not contain singular blocks at all. The generic canonical form of $n \times n$ T-palindromic pencils can be found in [6, Thm. 6]. For the remaining structures, the canonical form can be obtained by applying either the transformation $\lambda \mapsto-\lambda$ (for the $T$-antipalindromic structure, as in the proof of Theorem 4.2), the Cayley transformations $\mathcal{C}_{+1}$ and $\mathcal{C}_{-1}$ (for the $T$-even structure, as in the proof of Theorem 4.5), or these Cayley maps followed by reverting the coefficients $A_{0}$ and $A_{1}$ (for the T-odd structure, as in the proof of Theorem 4.6). We note that, though Theorem 6 in [6] is stated for strict equivalence (in terms of the KCF) instead of congruence, the generic canonical form (for congruence) can be obtained from that one by gathering each couple of blocks $\left(\lambda+\mu_{i}\right) \oplus\left(\lambda+1 / \mu_{i}\right)$ in the form $\left[\begin{array}{cc}0 & \lambda+\mu_{i} \\ 1+\lambda \mu_{i} & 0\end{array}\right]$.

As a consequence, the generic canonical form in the full-rank case has nothing to do with the generic canonical forms obtained in (4.1)-(4.2), (4.5)-(4.8), and (4.10)(4.11) for rank-defficient cases. Despite this fact, the formulas for the dimension of $\mathrm{Pal}_{r}, \mathrm{Apal}_{r}, \mathrm{Even}_{r}$, and $\mathrm{Odd}_{r}$ in Theorems 4.1, 4.2, 4.5, and 4.6, are still valid for $r=n$. To see this, note that by replacing $r=n$ in these formulas we end up, in all cases, with $n^{2}$, which is the dimension of the set of structred $n \times n$ pencils, for any of the structures considered.

It is also interesting to compare the results on the dimension of sets of low-rank structured pencils with the case of nonstructured pencils. If we denote by $\mathbb{P}_{r}$ the set of (unstructured) $n \times n$ matrix pencils with rank at most $r$, then, as mentioned before, we have

$$
\operatorname{dim} \mathrm{Pal}_{n}=\operatorname{dim} \mathrm{Apal}_{n}=\operatorname{dim} \mathrm{Even}_{n}=\operatorname{dim} \operatorname{Odd}_{n}=\frac{1}{2} \operatorname{dim} \mathbb{P}_{n}=n^{2} .
$$

One may wonder whether these identities are still true or not for $r<n$. The answer is given in the following corollary.

COROllary 4.8. The following identities hold, for $r \leq n$ :

$$
\begin{gathered}
\operatorname{dim} \mathrm{Pal}_{r}=\operatorname{dim} \mathrm{Apal}_{r}=\operatorname{dim} \mathrm{Even}_{r}=\operatorname{dim} \mathrm{Odd}_{r} \\
= \begin{cases}\frac{1}{2} \operatorname{dim} \mathbb{P}_{r} & \text { if } r \text { is even, } \\
\frac{1}{2} \operatorname{dim} \mathbb{P}_{r}-\frac{1}{2}(n-r) & \text { if } r \text { is odd. }\end{cases}
\end{gathered}
$$

Proof. The result is a direct consequence of the first claim in Theorems 4.1, 4.2, 4.5, and 4.6, together with the fact that $\operatorname{dim} \mathbb{P}_{r}=r(3 n-r)$ [5, Thm. 3.3].

The identity for the case $r$ odd in Corollary 4.8 may be surprising when compared with the case $r$ even. However, the differences between these two cases can be explained by looking at the generic forms provided in (4.1)-(4.2), (4.5)-(4.6) (for the 
palindromic structures) and (4.7)-(4.8), (4.10)-(4.11) (for the alternating structures). More precisely, in the case $r$ odd, the generic canonical form contains some regular part in all structures, whereas in the case $r$ even it consists entirely of singular blocks. The presence of such regular part imposes some additional restrictions which should lead one to expect some differences in the dimension count. Nonetheless, a full explanation of these particular differences would require one to analyze more in detail the algebraic restrictions imposed by the presence of these blocks, something which is beyond the scope of this paper.

5. Conclusions and future work. We have proved that the algebraic sets of $T$-palindromic, $T$-antipalindromic, $T$-even, and $T$-odd matrix pencils with rank at most $r<n$ are irreducible algebraic sets. This is in stark contrast with the case of $n \times n$ unstructured matrix pencils with rank at most $r$, which is an algebraic set with $r+1$ irreducible components. We have described these sets of structured matrix pencils with bounded rank as the closures of the congruence orbit of a certain structured pencil given in canonical form. As a consequence, we have determined the generic canonical form of structured pencils with rank at most $r$, for any of the previous structures. We have also computed the dimension of each of these sets.

A natural continuation of this work is to address the same questions for other structures arising usually in applications, like the Hermitian, skew-Hermitian, *palindromic, $*$-antipalindromic, or $*$-alternating structures. The sets of $n \times n$ structured pencils satisfying any of these structures are not algebraic sets over $\mathbb{C}$, but over $\mathbb{R}$, and for this reason we have not considered them here. Moreover, the description of low-rank pencils with these structures provided in [10] as a sum of rank-1 pencils suggests that the treatment of these structures deserves some additional effort.

Another possible line of research is to extend the results in the paper to matrix polynomials of higher degree. There are some recent contributions in this direction. In [11] the authors have described the generic scalar spectral information of arbitrary (nonstructured) matrix polynomials with bounded rank and fixed degree, and in [12] they have obtained an analogous description for the set of skew-symmetric matrix polynomials with bounded rank and fixed degree.

Acknowledgments. This work was done while the author was visiting the TU Berlin within the research group on Numerical Mathematics. The hospitality of the whole group is gratefully acknowledged. The author wishes to acknowledge fruitful and stimulating discussions with C. Mehl and V. Mehrmann. He also thanks J. M. Landsberg for insightful ideas that allowed him to prove Proposition 3.2, J. Brundan for pointing out reference [21], V. Muñoz for pointing out the notion of constructible sets, and two anonymous referees for the careful reading of the original manuscript and its revisions, and for very useful comments that led to a significant improvement.

\section{REFERENCES}

[1] L. BATZKe, Generic rank-one perturbations of structured regular matrix pencils, Linear Algebra Appl., 458 (2014), pp. 638-670.

[2] L. Batzke, Generic Low-Rank Perturbations of Structured Regular Matrix Pencils and Structured Matrices, Ph.D. thesis, TU Berlin, Berlin, 2015.

[3] F. De Terán, Canonical forms for congruence of matrices: A tribute to $H$. W. Turnbull and A. C. Aitken, SeMA J., 73 (2016), pp. 7-16.

[4] F. De TerÁn AND F. M. Dopico, Low rank perturbation of Kronecker structures without full rank, SIAM J. Matrix Anal. Appl., 29 (2007), pp. 496-529.

[5] F. De TerÁN AND F. M. Dopico, A note on generic Kronecker orbits of matrix pencils with fixed rank, SIAM J. Matrix Anal. Appl., 30 (2008), pp. 491-498. 
[6] F. De Terán And F. M. Dopico, The solution of the equation $X A+A X^{T}=0$ and its application to the theory of orbits, Linear Algebra Appl., 434 (2011), pp. 44-67.

[7] F. De Terán And F. M. Dopico, Generic change of the partial multiplicities of regular matrix pencils under low rank perturbations, SIAM J. Matrix Anal. Appl., 37 (2016), pp. 823-835.

[8] F. De Terán, F. M. Dopico, And J. M. Landsberg, An explicit description of the irreducible components of the set of matrix pencils with bounded normal rank, Linear Algebra Appl., 520 (2017), pp. 80-103.

[9] F. De Terán, F. M. Dopico, And J. Moro, Low rank perturbation of Weierstrass structure, SIAM J. Matrix Anal. Appl., 30 (2008), pp. 538-547.

[10] F. De Terán, C. Mehl, and V. Mehrmann, Low rank perturbation of regular structured matrix pencils, in preparation.

[11] A. Dmytryshyn And F. M. Dopico, Generic complete eigenstructures for sets of matrix polynomials with bounded rank and degree, Linear Algebra Appl., 535 (2017), pp. 213-230.

[12] A. Dmytryshyn And F. M. Dopico, Generic skew-symmetric matrix polynomials with fixed rank and fixed odd grade, Linear Algebra Appl., 536 (2018), pp. 1-18.

[13] A. DMytryshyn And B. KÅGSTRÖM, Orbit closure hierarchies for skew-symmetric matrix pencils, SIAM J. Matrix Anal. Appl., 35 (2014), pp. 1429-1443.

[14] A. Dmytryshyn, B. KÅgström, and V. V. Sergeichuk, Skew-symmetric matrix pencils: Codimension counts and the solution of a pair of matrix equations, Linear Algebra Appl., 438 (2013), pp. 3375-3396.

[15] A. Dmytryshyn, B. Kågström, and V. V. Sergeichuk, Symmetric matrix pencils: Codimension counts and the solution of a pair of matrix equations, Electron. J. Linear Algebra, 27 (2014), pp. 1-18.

[16] A. Edelman, E. Elmroth, and B. KÅgström, A geometric approach to perturbation theory of matrices and matrix pencils. Part I: Versal deformations, SIAM J. Matrix Anal. Appl., 18 (1997), pp. 653-692.

[17] A. Edelman, E. Elmroth, And B. KÅgström, A geometric approach to perturbation theory of matrices and matrix pencils. Part II: A stratification-enhanced staircase algorithm, SIAM J. Matrix Anal. Appl., 20 (1999), pp. 667-699.

[18] E. Eich-Soellner And C. Fuhrer, Numerical Methods in Multibody Dynamics, European Consortium for Mathematics in Industry, Teubner, Leipzig, 1998.

[19] F. R. Gantmacher, The Theory of Matrices, Chelsea, New York, 1959

[20] R. A. Horn and C. R. Johnson, Topics in Matrix Analysis, Cambridge University Press, Cambridge, 1994

[21] J. E. Humphreys, Linear Algebraic Groups, Springer-Verlag, Berlin, 1975.

[22] D. S. Mackey, N. Mackey, C. Mehl, and V. Mehrmann, Structured polynomial eigenvalue problems: good vibrations from good linearizations, SIAM J. Matrix Anal. Appl., 28 (2006), pp. 1029-1051.

[23] D. S. Mackey, N. Mackey, C. Mehl, and V. Mehrmann, Jordan structures of alternating matrix polynomials, Linear Algebra Appl., 432 (2010), pp. 867-891.

[24] C. Mehl, V. Mehrmann, and M. Wojtylak, On the distance to singularity via low rank perturbations, Oper. Matrices, 9 (2015), pp. 733-772.

[25] C. Mehl, V. Mehrmann, and M. Wojtylak, Parameter-dependent rank-one perturbations of singular Hermitian or symmetric pencils, SIAM J. Matrix Anal. Appl., 38 (2017), pp. $72-95$.

[26] D. Mumford, Algebraic Geometry (I), Springer-Verlag, Berlin, 1995.

[27] R. RiazA, Differential-Algebraic Systems: Analytical Aspects and Circuit Applications, World Scientific, Singapore, 2008.

[28] L. H. Rowen, Graduate Algebra: Noncommutative View, Grad. Stud. Math. 91, American Mathematical Society, Providence, 2006.

[29] C. SCHRÖDER, A structured Kronecker form for the palindromic eigenvalue problem, in PAMM Proc. Appl. Math. Mech., 6 (2006), pp. 721-722.

[30] I. R. Shafarevich, Basic Algebraic Geometry (I), 2nd ed., Springer-Verlag, Berlin, 1994. 